\title{
Study on adsorption and desorption of ammonia on graphene
}

\author{
Zhengwei Zhang, Xinfang Zhang, Wei Luo, Hang Yang, Yanlan He, Yixing Liu, Xueao Zhang and Gang Peng*
}

\begin{abstract}
The gas sensor based on pristine graphene with conductance type was studied theoretically and experimentally. The time response of conductance measurements showed a quickly and largely increased conductivity when the sensor was exposed to ammonia gas produced by a bubble system of ammonia water. However, the desorption process in vacuum took more than $1 \mathrm{~h}$ which indicated that there was a larger number of transferred carriers and a strong adsorption force between ammonia and graphene. The desorption time could be greatly shortened down to about 2 min by adding the flow of water-vapor-enriched air at the beginning of the recovery stage which had been confirmed as a rapid and high-efficiency desorption process. Moreover, the optimum geometries, adsorption energies, and the charge transfer number of the composite systems were studied with first-principle calculations. However, the theoretical results showed that the adsorption energy between $\mathrm{NH}_{3}$ and graphene was too small to fit for the experimental phenomenon, and there were few charges transferred between graphene and $\mathrm{NH}_{3}$ molecules, which was completely different from the experiment measurement. The adsorption energy between $\mathrm{NH}_{4}$ and graphene increased stage by stage which showed $\mathrm{NH}_{4}$ was a strong donor. The calculation suggested that $\mathrm{H}_{2} \mathrm{O}$ molecule could help a quick desorption of $\mathrm{NH}_{4}$ from graphene by converting $\mathrm{NH}_{4}$ to $\mathrm{NH}_{3}$ Or $\left(\mathrm{NH}_{3}\right) \mathrm{n}\left(\mathrm{H}_{2} \mathrm{O}\right) \mathrm{m}$ groups, which was consistent with the experimental results. This study demonstrates that the ammonia gas produced by a bubble system of ammonia water is mainly ammonium groups of $\mathrm{NH}_{3}$ and $\mathrm{NH}_{4}$, and the $\mathrm{NH}_{4}$ moleculars are ideal candidates for the molecular doping of graphene while the interaction between graphene and the $\mathrm{NH}_{3}$ moleculars is weak.
\end{abstract}

Keywords: Graphene gas sensor; DFT; Ammonia; Adsorption and desorption

\section{Background}

Ammonia detection has a great significance in many areas, such as environmental protection and industrial inspection. Furthermore, ammonia detection has good prospects in medicine diagnose. For instance, measurements of exhaled ammonia may differentiate between viral and bacterial infections in lung diseases to justify the use of antibiotics [1], and ammonia detection may be used to indirectly measure urea levels for renal disease monitoring [2]. Identifying these signaling metabolites (disease markers) and measuring them in trace concentrations is not a trivial problem, and the low concentrations of analyte molecules presents a major challenge, along with the specificity to a given analyte. Recently, low-dimensional materials used for gas detection has become a trend $[3,4]$,

\footnotetext{
* Correspondence: penggang@nudt.edu.cn

College of Science, National University of Defense Technology, Changsha 410073, China
}

it has been reported that it is possible to use graphene as a gas sensor with high sensitivity and high accuracy for detecting ammonia groups $[5,6]$. Graphene is considered to be an excellent kind of sensor material due to its following properties: (i) graphene is a single atomic layer of graphite with a larger specific area than any other materials, which maximizes the interaction between the surface dopants and the adsorbates; (ii) as a kind of special material with zero bandgap, graphene has a extremely low Johnson noise [7], for which a slight change of carrier concentration can cause a notable variation of electrical conductivity; (iii) graphene has limited crystal defects, which ensures a low level of excess noise caused by thermal switching [7]. F. Schedin et al.'s study showed that micrometer-sized sensors made from graphene were capable of detecting individual gases, and the study found out that the changes in graphene conductivity during chemical exposure were quantized, with each event signaling adsorption or desorption of a single

\section{苗 Springer}


$\mathrm{NH}_{3}$ molecule, and proved that $\mathrm{NH}_{3}$ was acting as a strong donor for graphene and the desorption was relatively difficult [5]. Hugo E Romero et al. considered that the slow $\mathrm{NH}_{3}$ desorption rate from supported graphene FETs was consistent with a Fickian diffusion process of molecules in the $\mathrm{SiO}_{2} /$ graphene interface [8]. Lakshman K. Randeniya et al. has developed a new method by adding the flow of water-vapor-enriched air at the beginning of the recovery stage to realize a rapid desorption, and researchers believe that shifts in the substrate defect states of graphene caused by the water adsorption can weaken the interaction between $\mathrm{NH}_{3}$ and defective graphene [9]. However, O. Leenaerts et al. investigated the adsorption of $\mathrm{NH}_{3}$ on graphene substrate with first-principle calculations, and the results illustrated a very small adsorption energy (31 meV) and a small charge transfer $(0.027 \pm 0.001$ electrons per molecule at pristine sites) from $\mathrm{NH}_{3}$ to graphene [6].

These explanations revealed the truth from some degree, but some of these explanations are still not theoretically grounded or lack experimental verifications. Therefore, it is hard to treat them as a fully complete conclusion. In this work, we studied the adsorption and desorption of ammonia groups on graphene theoretically and experimentally. Our study demonstrates that the interaction between ammonia groups $\mathrm{NH}_{4}$ and graphene is much stronger than that of ammonia groups $\mathrm{NH}_{3}$. We take the view that hydrogen atom plays an important role in the adsorption between $\mathrm{NH}_{3}$ and graphene, and we find that $\mathrm{H}_{2} \mathrm{O}$ helps $\mathrm{NH}_{4}$ to take off one hydrogen atom and achieves a rapid desorption from graphene sheets.

\section{Methods}

A pristine graphene was first prepared by mechanical exfoliation (repeated peeling) of small mesas of highly oriented pyrolytic graphite, which was then transferred to highly $\mathrm{p}++$ doped silicon wafer with a thermally formed $\mathrm{SiO}_{2}(300 \mathrm{~nm})$ layer working as the sensors' bottom gate through a dry transfer procedure $[10,11]$. The number of layers $(N)$ in a graphene film was determined with microRaman scattering using the shape of the 2D Raman band at $\sim 2700 \mathrm{~cm}^{-1}$ and the intensity of the Raman-active Gband scattering at $\sim 1585 \mathrm{~cm}^{-1}$ by a LabRAM XploRA with 532-nm excitation and low power which results was confirmed as a single-layer graphene. Then, the $\mathrm{Cr}(5 \mathrm{~nm})$ / $\mathrm{Au}(100 \mathrm{~nm})$ electrodes, used as the source and the drain electrodes of graphene-FET with channel width of $10 \mu \mathrm{m}$ (Fig. 1a), were fabricated by an e-beam lithography process followed by an e-beam metal deposition process. To conveniently measure the gas-sensing properties, the electrodes of the sensors were bonded to a custom socket with gold wires. The electrical conductance, transfer characteristic, and time response of the graphene FET sensor were measured with a Keithley 2400 sourcemeter and a voltage source $( \pm 100 \mathrm{~V})$. The sensing performances of as-

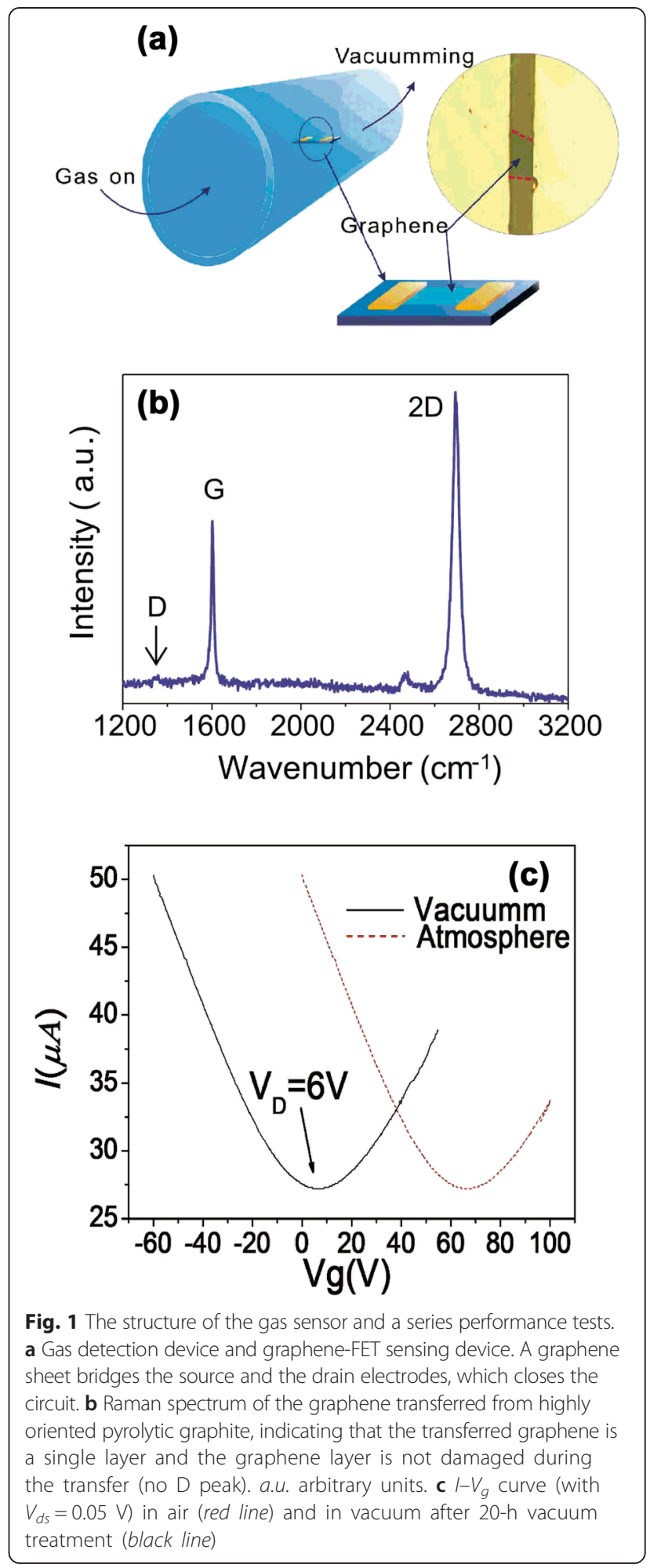

fabricated sensing devices were characterized under practical conditions (i.e., room temperature and atmospheric pressure) against low concentration of ammonia groups produced by a bubble system of ammonia water [9]. A sensor chamber with an electrical feed through was used 
to house the sensing device for gas-sensing characterizations (Fig. 1a). Variations in the electrical conductance of graphene sheets were monitored by simultaneously applying a low constant $\mathrm{DC}$ voltage $(0.05 \mathrm{~V})$ and recording the corresponding change in current passing through the device when the device was exposed periodically to $\mathrm{H}_{2} \mathrm{O}$ or $\mathrm{NH}_{3}$-laden high-purity argon and vacuum environment (ultimate vacuum, $\sim 1 \mathrm{~Pa}$ ). A sensing test cycle typically consists of three consecutive steps that include exposures of the device to (i) vacuum environment to record a base value of the sensor conductance, (ii) target gas to register a sensing signal, and (iii) vacuum environment for the sensor recovery. All the electrical measurements were carried out at room temperature. Five different sensors for the same process were measured which showed similar behaviors except the magnitude of conductance (the difference is less than $10 \%$ ) and each measurement was repeated for at least five times.

Theoretically, the density functional theory (DFT) calculations were performed with CASTEP [12]; the generalized gradient approximation (GGA) was used to deal with the exchange and correlation term. It is well known that the local density approximation (LDA) is normally inaccurate in describing the van der Waals-like interaction, and the advantage of GGA over LDA in this work is that the GGA will not lead to a strong bonding of the molecules as LDA does [13]. A plane wave basis set with a cutoff energy of $800 \mathrm{eV}$ and pseudopotentials of the Troullier-Martins type and non-spin-polarized calculations were used in this study. The total system consisted of a $3 \times 3$ graphene supercell (18 C atoms) with a single molecule adsorbed and a distance of $16 \AA$ between adjacent graphene layers. The Brillouin zone integration was performed within the Monkhorst-Pack scheme using $5 \times 5 \times$ $1 \mathrm{k}$ points [14]. For the calculation of the density of states (DOS), a $20 \times 20 \times 1$ Monkhorst-Pack grid and a Gaussian smearing of $0.05 \mathrm{eV}$ were used [15].

\section{Results and discussion}

The pristine graphene was prepared by mechanical exfoliation, and the Raman measurement was applied to study the layer numbers of graphene in room temperature under the atmosphere. In Fig. 1b, no D peak $\left(1350 \mathrm{~cm}^{-1}\right)$ was observed in graphene indicating that the quality of the graphene was maintained during the transfer, and the ratio of intensity $\mathrm{G}$ peak and $2 \mathrm{D}$ peaks was about 0.5 , which was a typical value of single-layer graphene. The basic electrical properties of bottom-gated bilayer graphene FETs were first studied in the atmospheric environment. We found that the Dirac peak was about $60 \mathrm{~V}\left(V_{D}=60 \mathrm{~V}\right)$ (Fig. 1c red line), which illustrated that this shift was attributed to an unintentional doping of the films by absorbing water and gas molecules or other impurity substance from the supporting substrate
$[16,17]$. Particularly, we found that the graphene film could return to the intrinsic state roughly after $20 \mathrm{~h}$ of vacuum treatment $(<1 \mathrm{~Pa})$, and then the Dirac peak was decreased to about $6 \mathrm{~V}\left(V_{D}=6 \mathrm{~V}\right)$. Prolonged vacuum treatment was capable of removing the adsorbed air molecules strongly. The total amount of effective charged impurities can be simply estimated by the amount of Dirac voltage shift, $V_{D}$, using the following expression [16]:

$$
N_{\text {imp }}=C_{g s} \times V_{D} / e
$$

where $C_{g s}$ is the capacitance of the $\mathrm{SiO}_{2}$ between the graphene and the silicon substrate (the typical value is $11.5 \mathrm{nF} / \mathrm{cm}^{2}$ for $300 \mathrm{~nm} \mathrm{SiO}$ in our case) and $e$ is the unit charge. From Fig. 1c, we can extract the Dirac voltage shift and then calculate the corresponding amount of charged impurities by expression (1). The density of charged impurities is as high as $4.3 \times 10^{12} / \mathrm{cm}^{-2}$ in the air, while that is about $0.43 \times 10^{12} / \mathrm{cm}^{-2}$ in the vacuum, with the corresponding Dirac voltage point decreased from 60 to $6 \mathrm{~V}$. Moreover, for each trace in Fig. 1c, we can extract the slope of linear region $\left(K_{h}\right.$ and $\left.K_{e}\right)$ from which one can extract the mobilities for holes (electrons) according to [18]:

$$
\begin{aligned}
\mu & =(L / W) \times 1 / C_{g s} / V_{S D} \times \frac{\partial I}{\partial V_{g}} \\
& =\frac{C \times K}{V_{S D}}
\end{aligned}
$$

where $C$ is a constant related to electrode geometry structure and dielectric material of the device, $K$ is the slope of linear region ( $K_{h}$ for hole, $K_{e}$ for electron), and

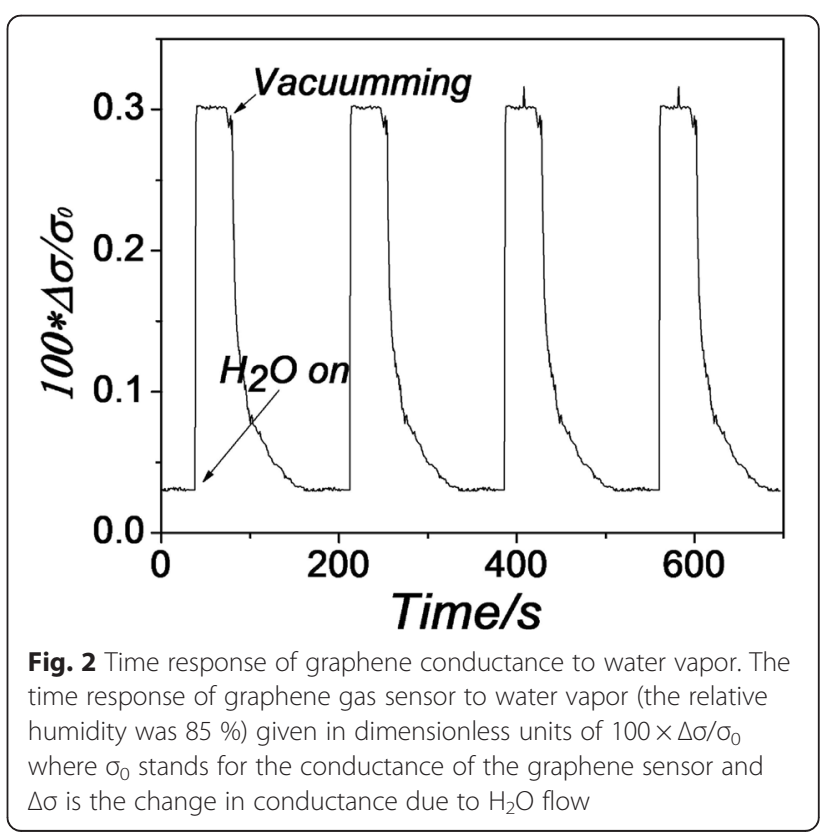



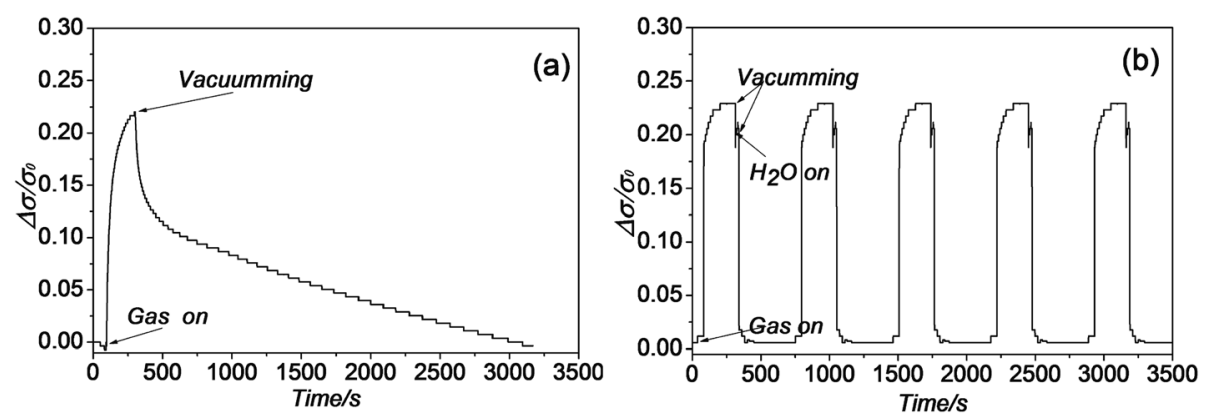

Fig. 3 Time response of graphene conductance to $5 \mathrm{ppm}$ ammonia in argon. a A single 2-min exposure and spontaneous recovery in vacuum treatment and $\mathbf{b}$ five consecutive 2-min exposures where water-vapor-enriched argon air was used to achieve quick desorption between exposures

$\mu$ is the mobility ( $\mu_{h}$ for hole and $\mu_{e}$ for electron). From the above expression, the mobilities of the hole and electron were almost same and which value was about $2000 \mathrm{~cm}^{2}(\mathrm{~V} \cdot \mathrm{S})^{-1}$.

The response of graphene gas sensor to high-purity argon (higher than $99.99 \%$ ) was first measured and there was no obvious change in conductance. Then, the time response of the sensor's conductance to a mixture gas (the relative humidity is $\sim 85 \%$ ) by diverting dry argon air through a water bubbler bottle was measured, and the results were shown in Fig. 2. Positive values of $\Delta \sigma$ were consistent with the theoretical and experimental studies which suggested $\mathrm{H}_{2} \mathrm{O}$ is an acceptor (hole doping) molecule [6]. When the flow of $\mathrm{H}_{2} \mathrm{O}$ was turned off and evacuated within about $5 \mathrm{~s}$, the conductance quickly decreased toward its baseline in 1 min, which illustrates that the force between graphene and water molecules is relatively weak [5].

The conductance of the gas sensor increased $~ 22.5 \%$ when exposed to $5 \mathrm{ppm}$ ammonia, shown in Fig. 3a. As is demonstrated in Fig. 3a, it took more than $1 \mathrm{~h}$ to reach the baseline value for the recovery stage. Fig. 3b shows a series of consecutive cycles where the recovery times were reduced to less than 2 min by adding the flow of water-vapor-enriched air at the beginning recovery stage as follows. When the ammonia gas was turned off and pumped out of chamber, water-vapor-enriched air was sucked into the chamber immediately and kept in the chamber for $1 \mathrm{~min}$, then a vacuum was used to desorb all the gas. The conductance changes and the (a)

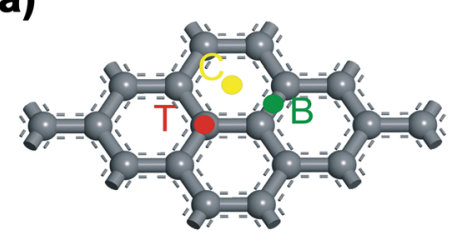

(c)

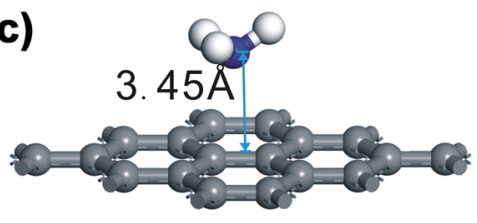

(e)

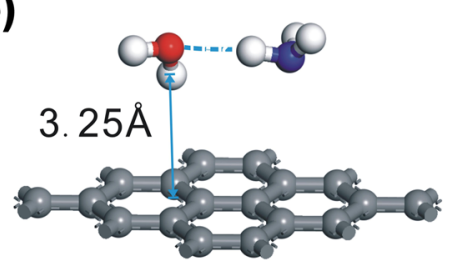

(b)

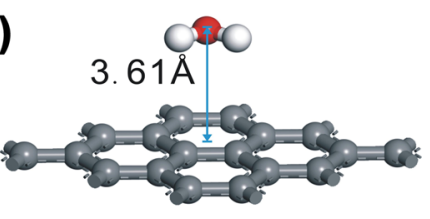

(d)

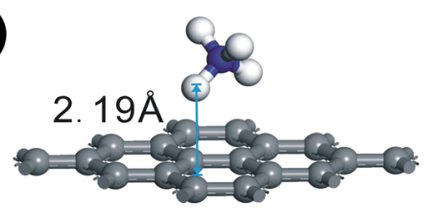

(f)

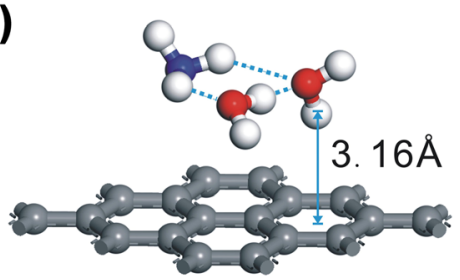

Fig. 4 Gas on graphene. Supercell $(3 \times 3)$ of graphene adsorbed all kinds of molecules and three adsorption sites are considered (a). Configuration of the $\mathrm{H}_{2} \mathrm{O}(\mathbf{b}), \mathrm{NH}_{3}(\mathbf{c}), \mathrm{NH} 4(\mathbf{d}), \mathrm{NH}_{3} \cdot \mathrm{H}_{2} \mathrm{O}(\mathbf{e})$, and $\mathrm{NH} 3 \cdot 2 \mathrm{H}_{2} \mathrm{O}(\mathbf{f})$ molecular on graphene sheets, all of the structures after optimization 
response shape profiles were extremely reproducible. These results confirmed the complete and rapid removal of ammonia from graphene sheets by water vapor [9].

Theoretical calculations on isolated defect-free graphene predicted that $\mathrm{NH}_{3}$ molecules should physisorb to the film without significant change of the band structure, and only a slight charge transfer of $0.03-0.04$ e per adsorbed $\mathrm{NH}_{3}$ molecule was predicted to occur [6]. It seems that theoretical calculations are inconsistent with our experiment results. To fully clarify the mechanism of the adsorption and desorption between ammonia and graphene sheets, it is important to understand the interactions between the graphene surface and the adsorbate molecules. The firstprinciple calculations were performed based on DFT which has been successfully applied to study the molecular adsorbates on single-walled (carbon) nanotubes (SWNT) and graphene sheets $[6,19]$. In our calculation, the molecules were first placed in high symmetry positions ( $\mathrm{C}, \mathrm{B}$, and $\mathrm{T}$ sites) and then relaxed [6]. The configurations of the molecule-graphene system are shown in Fig. 4. Generally, larger absolute value of the adsorption energy $\left(E_{a}\right)$ means stronger binding between molecule and graphene. The $E_{a}$ between the gas molecule and graphene is defined as

$$
E_{a}=E_{\text {system }}-E_{\text {graphene }}-n E_{\text {molecule }}
$$

where $E_{\text {system }}$ is the total energy of the optimized equilibrium configuration of the graphene and the adatom; $E_{\text {graphene }}$ is the total energy of the isolated graphene; $E_{\text {mo- }}$ lecule is the total energy of the corresponding adsorption molecules in its ground state; $n$ is the number of gas molecules in the system.

The following orientations of the molecule with respect to the graphene surface was studied in our calculations, for $\mathrm{H}_{2} \mathrm{O}$ molecule starting from the $\mathrm{O}$ atom, the $\mathrm{H}-\mathrm{O}$ bonds pointing parallel to the graphene surface $(\mathrm{P})$ [5], and two orientations of the ammonia molecule were investigated, one with the $\mathrm{H}$ atoms pointing away from the surface $(\mathrm{U})$ and the other with the $\mathrm{H}$ atoms pointing to the surface (D). The calculation results for the optimized structures were summarized in Tables 1 and 2 . From Table 1, the adsorption energy was about 26$29 \mathrm{meV}$ per $\mathrm{H}_{2} \mathrm{O}$-graphene group for different $\mathrm{H}_{2} \mathrm{O}$ positions with sheet-adsorbate distances of $3.6 \AA$ (Fig. 4b), and only 0.01-0.02 e charges were transferred from

Table 1 The adsorption energy $E_{a}$ and the charge transfer $\Delta Q$ from $\mathrm{H}_{2} \mathrm{O}$ to graphene with three different geometries

\begin{tabular}{llccl}
\hline Position & Orientation & Distance/ $\AA$ & $E_{a}\left(\mathrm{H}_{2} \mathrm{O}\right) / \mathrm{meV}$ & $\Delta Q$ \\
\hline $\mathrm{B}$ & $\mathrm{P}$ & 3.62 & 26.49 & $-0.01 \mathrm{e}$ \\
$\mathrm{C}$ & $\mathrm{P}$ & 3.61 & 29.32 & $-0.02 \mathrm{e}$ \\
$\mathrm{T}$ & $\mathrm{P}$ & 3.61 & 26.54 & $-0.01 \mathrm{e}$ \\
\hline
\end{tabular}

Table 2 The adsorption energy $E_{a}$ and the charge transfer $\Delta Q$ from $\mathrm{NH}_{3}$ to graphene with six different geometries

\begin{tabular}{llccc}
\hline Position & Orientation & Distance $/ \AA$ & $E_{a}\left(\mathrm{NH}_{3}\right) / \mathrm{meV}$ & $\Delta Q$ \\
\hline B & $\mathrm{U}$ & 3.40 & 31.44 & $0.02 \mathrm{e}$ \\
C & $\mathrm{U}$ & 3.49 & 45.53 & $0.02 \mathrm{e}$ \\
T & $\mathrm{U}$ & 3.66 & 38.04 & $0.02 \mathrm{e}$ \\
B & $\mathrm{D}$ & 3.46 & 39.45 & $-0.01 \mathrm{e}$ \\
C & $\mathrm{D}$ & 3.45 & 48.87 & $-0.01 \mathrm{e}$ \\
$\mathrm{T}$ & $\mathrm{D}$ & 3.45 & 39.42 & $-0.01 \mathrm{e}$ \\
\hline
\end{tabular}

graphene to the $\mathrm{H}_{2} \mathrm{O}$ molecule. The results suggest that $\mathrm{H}_{2} \mathrm{O}$ acts as an acceptor on graphene which is in accordance with our experiment. From Table 2, the adsorption energy in $\mathrm{NH}_{3}$-graphene group was small $(31-45 \mathrm{meV}$ ) as well as that in $\mathrm{H}_{2} \mathrm{O}$-graphene group, and the charge transfer, however, was solely determined by the orientation of the $\mathrm{NH}_{3}$ molecule. There was a slight charge transfer of $0.02 \mathrm{e}$ from the molecule to the graphene surface in the $\mathrm{U}$ orientation and a extremely slight charge transfer $(0.01 \mathrm{e})$ from graphene to $\mathrm{NH}_{3}$ molecule in the $\mathrm{D}$ orientation, and the bottom atom of the physisorbed molecule was located 3.4-3.6 ̊ (Fig. 4c) above the graphene layer. The calculation results indicated weak adsorption ability between $\mathrm{NH}_{3}$ molecule and graphene in both orientations, which is difficult to explain the strong adsorption and the donor character as observed experimentally.

These results were in agreement with those already reported in previous studies on the binding energies of physisorbed $\mathrm{NH}_{3}$ molecules on graphene [5, 6]. All of these results showed that ammonia molecules $\mathrm{NH}_{3}$ are not good candidates for effective molecular doping of graphene. Obviously, there are other chemical groups that physisorb more strongly on graphene sheets and shift the Fermi energy inside the valence or conduction bands. Bradley et al. found that vacuum-degassed SWNT-FETs were insensitive to $\mathrm{NH}_{3}$; they suggested that to detect $\mathrm{NH}_{3}$ gas in the FET response, one should dissolve the $\mathrm{NH}_{3}$ in a $\mathrm{H}_{2} \mathrm{O}$ monolayer which forms on the nanotube FETs under ambient lab conditions. They proposed that the $\mathrm{NH}_{3}$ molecule in this environment could become a cation with the charge compensated from the SWNTs, i.e., acting as an $n$-dopant [20]. In fact, hydrogen is an ordinary impurity in electronic devices [21, 22], playing an important role in many reactions between physisorbed $\mathrm{NH}_{3}$ molecules and hydrogen adatoms to generate

Table 3 The adsorption energy $E_{a}$ and the charge transfer $\Delta Q$ from $\mathrm{NH}_{4}$ to graphene with three different geometries

\begin{tabular}{lccl}
\hline Position & Distance/A & $E_{a}\left(\mathrm{NH}_{4}\right) / \mathrm{meV}$ & $\Delta Q$ \\
\hline B & 2.86 & 528.06 & $0.19 \mathrm{e}$ \\
$\mathrm{C}$ & 2.19 & 644.29 & $0.54 \mathrm{e}$ \\
$\mathrm{T}$ & 2.21 & 628.94 & $0.34 \mathrm{e}$ \\
\hline
\end{tabular}


Table 4 The adsorption energy $E_{a}$ and the charge transfer $\Delta Q$ from $\mathrm{NH}_{3} \cdot \mathrm{H}_{2} \mathrm{O}$ and $\mathrm{NH}_{3} \cdot 2 \mathrm{H}_{2} \mathrm{O}$ to graphene

\begin{tabular}{lll}
\hline Distance/ $\AA$ & $E_{a}\left(\mathrm{NH}_{3} \cdot \mathrm{H}_{2} \mathrm{O}\right) / \mathrm{meV}$ & $\Delta Q$ \\
3.25 & 43.70 & $0.01 \mathrm{e}$ \\
Distance/ $\AA$ & $E_{a}\left(\mathrm{NH}_{3} \cdot 2 \mathrm{H}_{2} \mathrm{O}\right) / \mathrm{meV}$ & $\Delta Q$ \\
3.16 & 73.52 & $-0.01 \mathrm{e}$ \\
\hline
\end{tabular}

chemical species that have stronger interactions with the graphene layer. Specifically, an isolated $\mathrm{H}$ impurity on graphene can be captured by an $\mathrm{NH}_{3}$ molecule in an exothermic reaction that releases $1.00 \mathrm{eV}$ of energy [15].

Furthermore, results for physisorbed $\mathrm{NH}_{4}$ species on a defect-free graphene layer was calculated in different graphene positions and a special orientation [15] (two of the $\mathrm{NH}_{4}$ hydrogen atoms pointing toward the graphene sheets, while the other two hydrogen species are on the other side of the ammonium group, see Fig. 4d). From Table 3, we noticed that the $\mathrm{NH}_{4}$ molecule were adsorbed on graphene in a stronger manner, and the interaction between dangling bonds of graphene and $\mathrm{NH}_{4}$ was very strong and partial electron transfer occurs from graphene. The adsorption energy was 528-644 meV which was much larger than that of $\mathrm{NH}_{3}$ and $\mathrm{H}_{2} \mathrm{O}$ adsorbed in graphene sheets, and a huge charge transfer (0.19-0.54 e) from $\mathrm{NH}_{4}$ to graphene proved that physisorbed $\mathrm{NH}_{4}$ acted as a stronger donor for the basal plane of graphene, and the bottom atom of the $\mathrm{NH}_{4}$ was located $2.19 \AA$ (Fig. 4d) above the graphene layer. There was a tight connection between them, and the results were in accordance with our experiment results.

It is well known that there exists complicated chemical equilibrium in ammonia-water mixtures, and the presence of $\mathrm{NH}_{4}^{+}$and $\mathrm{OH}^{-}$ions in water solutions is a wellestablished fact that meets the following reaction:

$$
\mathrm{NH}_{3}+\mathrm{H}_{2} \mathrm{O} \Leftrightarrow \mathrm{NH}_{4}^{+}+\mathrm{OH}^{-} \mathrm{K}_{\mathrm{b}}=\frac{\left[\mathrm{NH}_{4}^{+}\right]\left[\mathrm{OH}^{-}\right]}{\left[\mathrm{NH}_{3}\right]}
$$

and the experimental equilibrium constant $K_{b}$ for this reaction at room temperature is $1.77 \times 10^{-5}$; therefore, the concentration of $\mathrm{NH}_{4}^{+}$and $\mathrm{OH}^{-}$in water solutions, although extremely small, is about $4.21 \times 10^{-3} \mathrm{~mol} / \mathrm{dm}^{3}$.

In fact, ammonia water exists in the form of clusters by hydrogen bonding [23]. Up to now, among all the possibilities, the only two which are experimentally supported are $\mathrm{NH}_{3} \cdot \mathrm{H}_{2} \mathrm{O}$ and $\mathrm{NH}_{3} \cdot 2 \mathrm{H}_{2} \mathrm{O}$ [24]. We chose these two typical groups for the calculation. Table 4 shows the construction of the clusters adsorption on the graphene sheets, and the orientations of the groups are parallel to the graphene surface (shown in Fig. 4e, f). Ignoring the adsorption site, we can find the adsorption energy is $43-73 \mathrm{meV}$, which is lower than that of $\mathrm{NH}_{4}$ adsorbed in graphene with a reduction of one order of

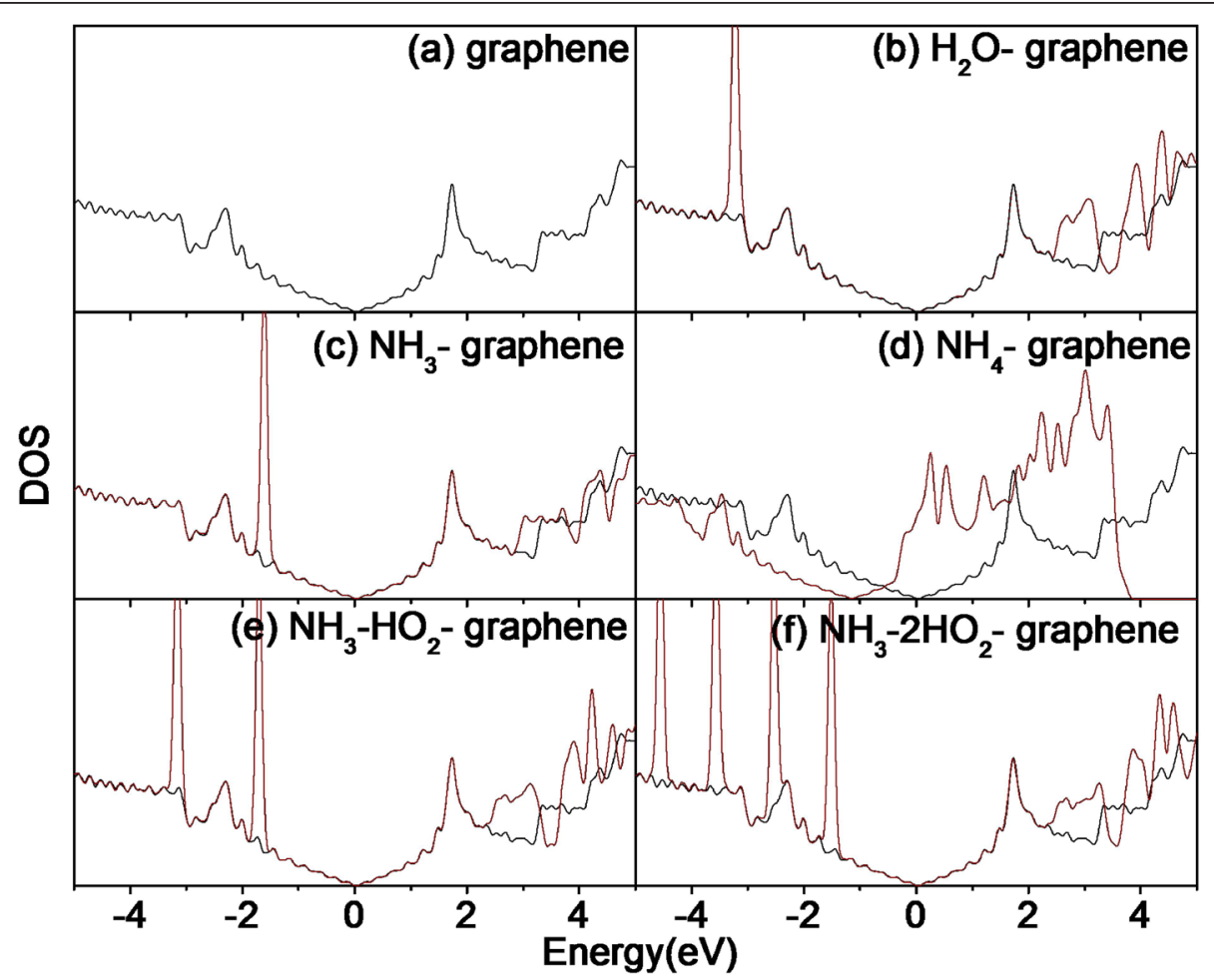

Fig. 5 The DOS of the gas-graphene system. Electronic density of state (DOS) of intrinsic graphene (a) and the gas adsorbed on graphene (b-f, red line is the DOS of the gas-graphene system) 
magnitude, and the charge transfer from clusters to graphene sheets is close to zero. The distance between the cluster and the graphene layer is about 3.16-3.25 (Fig. 4d), which illustrates that the ammonia-water groups have weak adsorption with graphene sheet, and that they can be desorbed from graphene easily.

To further determine the effects of gas absorption on electrical conductivity, the electronic densities of state (DOS) for all of the systems are calculated in Fig. 5. Figure $5 \mathrm{~b}$, c shows that the DOS of $\mathrm{H}_{2} \mathrm{O}$-graphene system and $\mathrm{NH}_{3}$-graphene system at the Fermi level changes little between molecules and graphene alone, resulting from the weak van der Waals interactions and the small charge transfer. The data depicted in Fig. $5 \mathrm{~d}$ demonstrate that reaction with $\mathrm{NH}_{4}$ is an effective way of molecular doping of graphene. The attachment of one $\mathrm{NH}_{4}$ group per 18 carbon atoms gives rise to a shift of the DOS so that the Fermi level of the $\mathrm{NH}_{4}$-laden system lies within the conduction band. In other words, $\mathrm{NH}_{4}$ physisorbed impurities act as strong donors for the basal plane of graphene. Figure 5e, $\mathrm{f}$ shows the group of $\mathrm{NH}_{3} \cdot \mathrm{H}_{2} \mathrm{O}$ and $\mathrm{NH}_{3} \cdot 2 \mathrm{H}_{2} \mathrm{O}$ interacting with graphene sheets with weak force, which proves that the clusters desorption is easier than that of $\mathrm{NH}_{4}$ group from graphene films.

\section{Conclusions}

In conclusion, the adsorptions of ammonia on graphene were investigated with experiment and first-principle calculation. The study demonstrates that the remarkable variation of the electrical conductivity is induced by the ammonia adsorption, and that the graphene gas sensor possesses an excellent characteristic of high sensitivity for ammonia gas detection. It was found experimentally that ammonia molecules produced by a bubble system of ammonia water were strongly adsorbed onto the graphene sheets; however, ammonia molecules were only weakly adsorbed onto the intrinsic graphene with small binding energy value and large distance between the $\mathrm{NH}_{3}$ molecules and graphene from first-principle calculations. The electronic structure and electrical conductivity of the intrinsic graphene have limited changes due to the adsorption of $\mathrm{NH}_{3}$ molecules. Moreover, this study found that the ammonium $\left(\mathrm{NH}_{4}\right)$ had strong interactions with graphene, forming a strong bond that introduces a large amount of shallow donor states into the system. When adding water molecules into the desorption process, the whole desorption process was greatly accelerated. We considered that ammonium molecules and water molecules generated the ammonia-water cluster and found that the cluster has weak adsorption with graphene sheets by calculation. The results are in accordance with the experiment. In a word, this study demonstrated that the ammonia gas produced by a bubble system of ammonia water were mainly molecular groups of $\mathrm{NH}_{3}$ and $\mathrm{NH}_{4}$, and the $\mathrm{NH}_{4}$ moleculars are ideal candidates for the molecular doping of graphene. However, the $\mathrm{NH}_{3}$ moleculars have weak interaction with graphene.

\section{Competing interests}

The authors declare that they have no competing interests.

\section{Authors' contributions}

ZWZ, XFZ, and WL carried out the gas sensor device and the measurements, the data analysis, and calculations. YXL and GP constructed the simulation model and drafted most of the manuscript. YLH and XAZ helped revise the manuscript. All authors read and approved the final manuscript.

\section{Acknowledgements}

The authors are grateful to Natural Science Foundation of China (No. 11104348), School Pre-research of National University of Defense Technology (JC11-02-08) for the financial support to this work. The calculations were performed on computers in the High Performance Computing Center of Central South University, China.

Received: 8 July 2015 Accepted: 27 August 2015

Published online: 16 September 2015

\section{References}

1. Kharitonov SA. Biomarkers of some pulmonary diseases in exhaled breath. Biomarkers. 2002;7:1-32.

2. Sawicka K, Gouma P, Simon S. Electrospun biocomposite nanofibers for urea biosensing. Sensors Actuators B Chem. 2005;108:585-8.

3. Srivastava A, Bhat C, Jain SK, Mishra PK, Brajpuriya R. Electronic transport properties of BN sheet on adsorption of ammonia $\left(\mathrm{NH}_{3}\right)$ gas. J Mol Model. 2015;21(3):1-8

4. Khan MS, Srivastava A, Chaurasiya R, Khan MS, Dua P. $\mathrm{NH}_{3}$ and $\mathrm{PH}_{3}$ adsorption through single walled $\mathrm{ZnS}$ nanotube: First principle insight. Chemical Physics Letters. 2015;636:103-9.

5. Schedin F, Geim A, Morozov S, Hill E, Blake P, Katsnelson M, et al. Detection of individual gas molecules adsorbed on graphene. Nat Mater. 2007;6(9):652-5.

6. Leenaerts $\mathrm{O}$, Partoens $\mathrm{B}$, Peeters $\mathrm{F}$. Adsorption of $\mathrm{H}_{2} \mathrm{O}, \mathrm{NH}_{3}, \mathrm{CO}, \mathrm{NO}_{2}$, and NO on graphene: a first-principles study. Physical Review B. 2008;77(12):125416.

7. Dutta P, Horn PM. Low-frequency fluctuations in solids: $1 F$ noise. Rev Mod Phys. 1981;53(3):497-516.

8. Romero HE, Joshi P, Gupta AK, Gutierrez HR, Cole MW, Tadigadapa SA, et al. Adsorption of ammonia on graphene. Nanotechnology. 2009;20(24):10830-2.

9. Randeniya LK, Shi H, Barnard AS, Fang J, Martin PJ, Ostrikov KK. Harnessing the influence of reactive edges and defects of graphene substrates for achieving complete cycle of room-temperature molecular sensing. Small. 2013;9(23):399-3999.

10. Dean C, Young A, Meric I, Lee C, Wang L, Sorgenfrei S, et al. Boron nitride substrates for high-quality graphene electronics. Nat Nanotechnol. 2010;5(10):722-6.

11. Peng $G$, Tisch $U$, Haick $H$. Detection of nonpolar molecules by means of carrier scattering in random networks of carbon nanotubes: toward diagnosis of diseases via breath samples. Nano Lett. 2009;9(4):1362-8.

12. Milman V, Winkler B, White J, Pickard C, Payne M, Akhmatskaya E, et al. Electronic structure, properties, and phase stability of inorganic crystals: A pseudopotential plane-wave study. Int J Quantum Chem. 2000;77(5):895-910.

13. Troullier, N. and J.L. Martins, Efficient pseudopotentials for plane-wave calculations. Physical Review B, 1991;43(3):1993.

14. Monkhorst HJ, Pack JD. Special points for Brillouin-zone integrations Physical Review B. 1976;13(12):5188.

15. Tsetseris $L$, Pantelides ST. Molecular doping of graphene with ammonium groups. Physrevb. 2012;85(15):543-8.

16. Chen J-H, Jang C, Adam S, Fuhrer M, Williams E, Ishigami M. Chargedimpurity scattering in graphene. Nat Phys. 2008;4(5):377-81.

17. Rumyantsev S, Liu G, Stillman W, Shur M, Balandin A. Electrical and noise characteristics of graphene field-effect transistors: ambient effects, noise sources and physical mechanisms. J Phys Condens Matter. 2010;22(39):395302. 
18. Kim BJ, Jang $\mathrm{H}$, Lee $\mathrm{S}-\mathrm{K}$, Hong BH, Ahn J-H, Cho JH. High-performance flexible graphene field effect transistors with lon Gel gate dielectrics. Nano Lett. 2010;10(9):3464-6.

19. Santucci S, Picozzi S, Di Gregorio F, Lozzi L, Cantalini C, Valentini L, et al. $\mathrm{NO}_{2}$ and $\mathrm{CO}$ gas adsorption on carbon nanotubes: experiment and theory. J Chem Phys. 2003;1 19(20):10904-10.

20. Bradley K, Gabriel J-CP, Briman M, Star A, Grüner G. Charge transfer from ammonia physisorbed on nanotubes. Physrevlett. 2003;91(21):218301.

21. Pantelides ST, Tsetseris L, Rashkeev S, Zhou X, Fleetwood DM, Schrimpf RD. Hydrogen in MOSFETs - a primary agent of reliability issues. Microelectronics Reliability. 2007:47(6):903-12.

22. Tsetseris L, Fleetwood D, Schrimpf R, Zhou X, Batyrev I, Pantelides S. Hydrogen effects in MOS devices. Microelectron Eng. 2007:84(910):2344-9.

23. Hoyer E. Ionisation Constants of Inorganic Acids and Bases in Aqueous Solution: Zusammengestellt von DD Perrin; Oxford, New York, Toronto, Sidney, Paris, Frankfurt; Pergamon Press 1982; 2. Auflage, 180Seiten; Pappb. $\$ 50,00$. Zeitschrift für Chemie. 1983;23(7):276.

24. Bell R. The Proton in Chemistry, 1973. London: Chapman and Hall; 1973.

\section{Submit your manuscript to a SpringerOpen ${ }^{\circ}$ journal and benefit from:}

- Convenient online submission

- Rigorous peer review

- Immediate publication on acceptance

- Open access: articles freely available online

- High visibility within the field

- Retaining the copyright to your article

Submit your next manuscript at $\gg$ springeropen.com 